
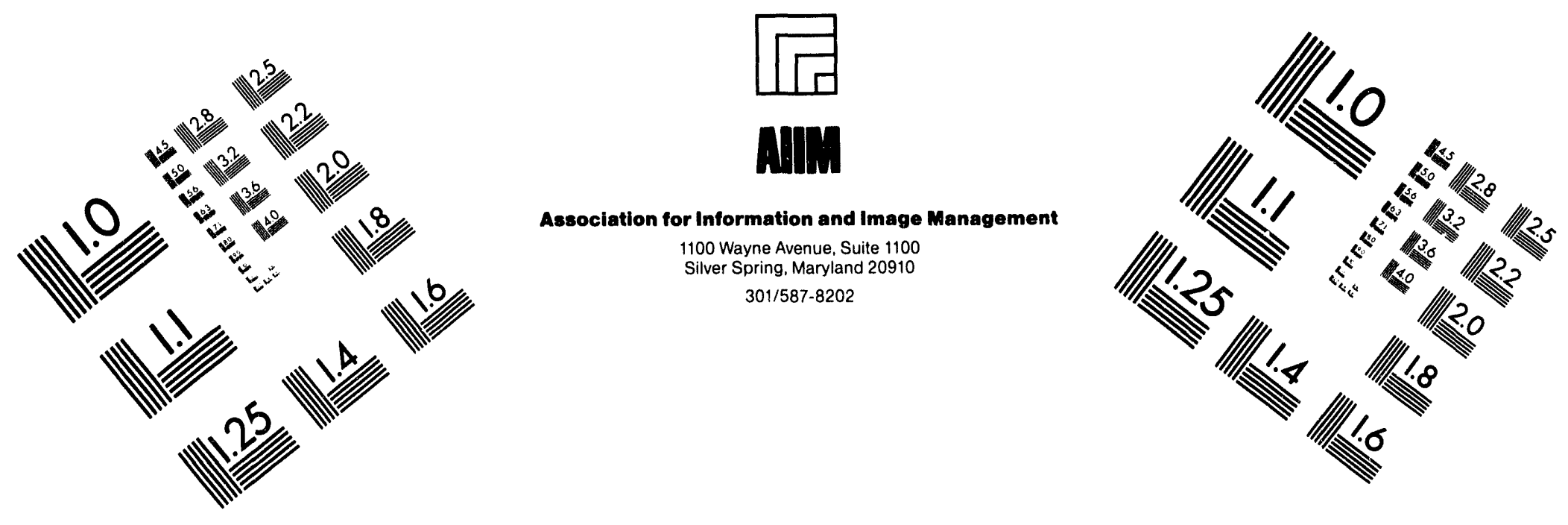

\title{
Centimeter
}

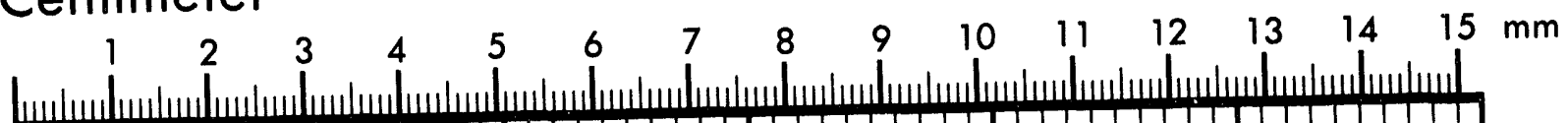

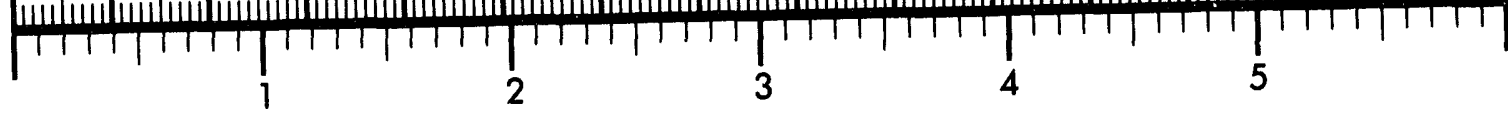
Inches
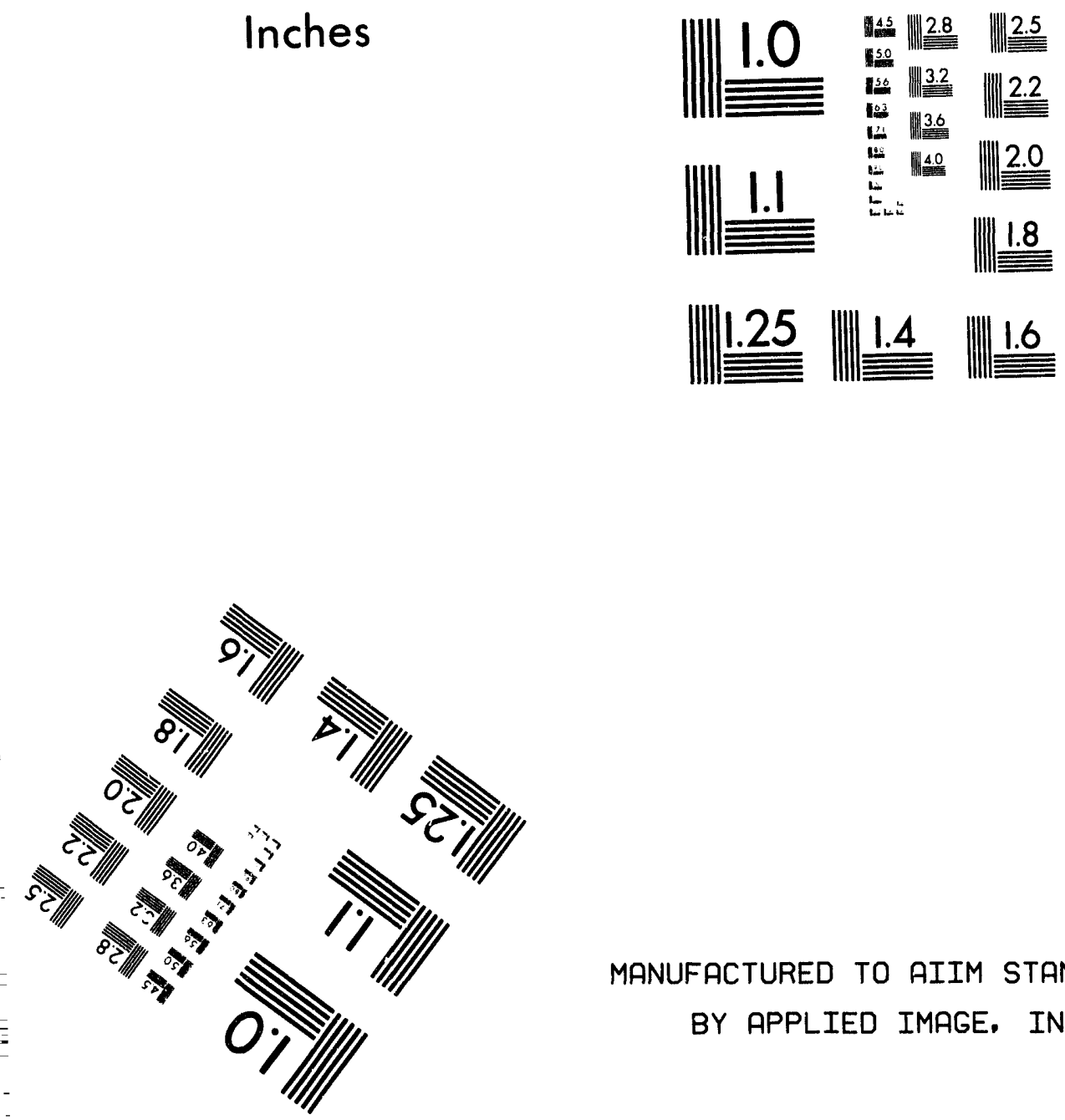

MANUFACTURED TO AIIM STANDARDS BY APPLIED IMAGE. INC.

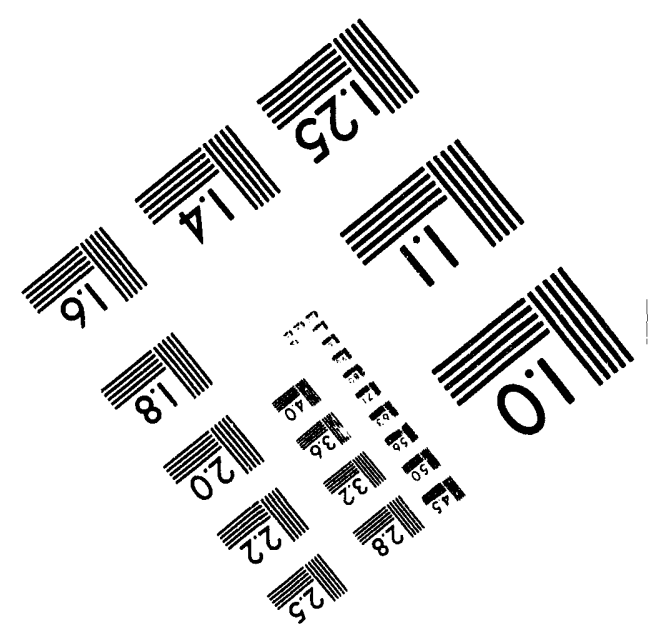



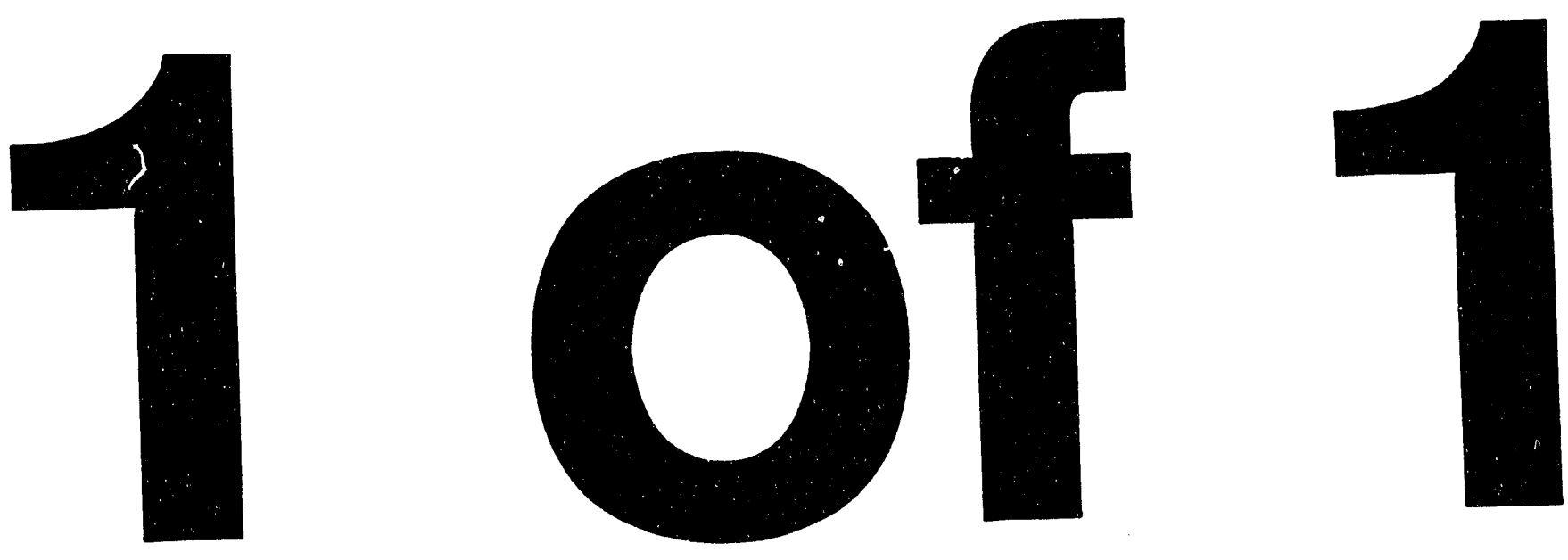
This report was prepared as an account of work sponsored by an agency of the United States Government. Neither the United States Government nor any agency thereof, nor any of their employees, makes any warranty, express or implied, or assumes any legal liability or responsibility for the accuracy, completeness, or usefulness of any information, apparatus, product, or process disclosed, or represents that its use would not infringe privately owned rights. Reference herein to any specific commercial product, process, or service by trade name, trademark, manufacturer, or otherwise does not necessarily constitute or imply its endorsement, recommendation, or favoring by the United States Government or any agency thereof. The views and opinions of authors expressed herein do not necessarily state or reflect those of the United States Government or any agency thereof.

\section{SCINTILLATING GLASS FIBER-OPTIC NEUTRON SENSORS}

M. Bliss et al

April 1994

Presented at the

1994 Spring Meeting

April 1994

San Francisco, California

Prepared for

the U.S. Department of Energy

under Contract DE-AC06-76RLO 1830

Pacific Northwest Laboratory

Richland, Washington 99352 


\title{
SCINTILLATING GLASS FIBER-OPTIC NEUTRON SENSORS
}

\author{
K.H. ABEL*, R.J. ARTHUR*, M. BLISS*, D.W. BRITE*, R.L. BRODZINSKI*, \\ R.A. CRAIG*, B.D. GEELHOOD*, D.S. GOLDMAN*, J.W. GRIFFIN*, R.W. PERKINS*, \\ P.L. REEDER*, W.C. RICHEY*, K A. STAHL*, D.S. SUNBERG*, R.A. WARNER*, \\ M. J. WEBER **, AND N.A. WOGMAN* \\ *Pacific Northwest Laboratory, Box 999, Richland, WA 99352 \\ **Lawrence Livermore National Laboratory, Box 808, Livermore, CA 94551
}

\begin{abstract}
Pacific Northwest Laboratory (PNL) has fabricated cerium-activated lithium silicate scintillating fibers via a hot-downdraw process. These fibers, which, as produced, typically have a transmission length (e-1 length) of greater than 2 meters, are found to undergo aging when subjected to room air. The aging, which is complete in a few weeks, reduces the transmission length to the order of 0.5 meter. Because of the high alkali content of the glass (on the order of 2030 mole \% lithia), we have attributed this aging to aqueous corrosion at the polymer cladding/glass interface. Changes in transmission with chemical treatment of the surface support the corrosion model. Fiber transmission performance has been preserved by modifying the hot-downdraw to a double crucible to produce glass-on-glass waveguides.
\end{abstract}

\section{INTRODUCTION}

The development of neutron-sensitive scintillating fibers was initiated to produce large-area, fast, solid-state, thermal-neutron detector packages which are insensitive to gamma rays. Based on experience and literature data, 1 it was determined that cerium-activated lithium silicate glass fiber waveguides would be the best candidate for the active part of the detector packages. Because such fibers are not available commercially, a fiber fabrication facility was developed.

As recently as 1993, cerium-activated lithium silicate fibers were described as having "...useful lengths of fiber [limited] to $<10 \mathrm{~cm}$." 1 By developing glass compositions specifically for fiber drawing, using strict oxidation state controls and rapid quenching, fiber with useful lengths in excess of $200 \mathrm{~cm}$ can be produced.

Because $\mathrm{Ce}^{4+}$ absorbs the light emitted by the $\mathrm{Ce}^{3+}$, the fibers are manufactured under reducing conditions to minimize $\mathrm{Ce}^{4+}$ production. The use of a hot downdraw system to produce the fiber avoids crystallization of the lithium-rich glass.

Figure 1 shows pulse-height spectra of radiation-induced events detected by a fiber ribbon for neutron and for gamma excitation. It is seen that neutron events produce a broad peak centered at channel 55 which gamma events do not. Gamma-induced Compton electrons produce much smaller pulses because the range of the electron is much greater than the diameter of the fiber. To enhance the discrimination against gammas, PNL flat detector panels are fabricated with photomultiplier tubes (PMTs) on both ends. Sum and coincidence requirements in such configurations give a counting efficiency ratio for neutrons, relative to gammas, of greater than 85,000 when at least 2 photoelectrons are required at each end of the panel. In practice, for complete detectors, most of the gamma sensitivity comes from the region near the PMT at which the fibers are bundled. The added thickness of the bundle region increases the probability that a gamma will result in a valid count. Complete detectors with a coincidence-plus-a-sum requirement 
of 4 or more photoelectrons have given neutron-efficiency-to-gamma-efficiency ratios as high as 8000 .

\section{FABRICATION PROCESS}

The fibers are fabricated using a hot downdraw tower. The bushing is heated in a controlledatmosphere induction furnace. A cold finger seals the tip of the bushing until the glass has been fined and is ready to draw. The fiber draw is started by removing the cold finger and then pulling a gob of glass off the bushing tip. When the fiber draw is established and stabilized, a cladding cup is placed around the fiber and a flow of cladding material is begun. The cladding material of choice is a thermally curing silicone resin. ${ }^{a}$ The silicone serves both as an optical cladding and as a mechanical buffer.

It is important to note that the use of an induction furnace is unusual. Most hot downdraw furnaces are resistively heated. An induction furnace facilitates the use of reducing atmospheres and the use of gas over-pressure to control the fiber diameter.

The transmission length ( $\mathrm{e}^{-1}$ distance) of the fiber is routinely measured using UV fluorescence excitation (Figure 2). Fluorescence in the fiber is excited at several points from the end of the fiber. The trapped fluorescence is input into a spectrometerb that has been modified for the high numerical aperture of the fiber. The spectrum is integrated from 375 to $450 \mathrm{~nm}$.

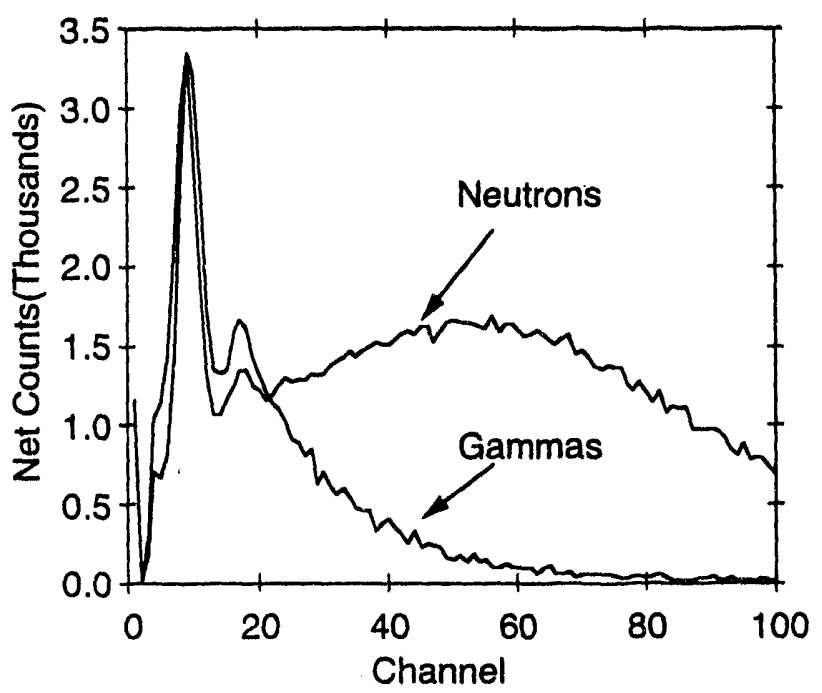

Figure 1. Pulse-height spectrum for a scintillating glass fiber ribbon under neutron and gamma excitation.

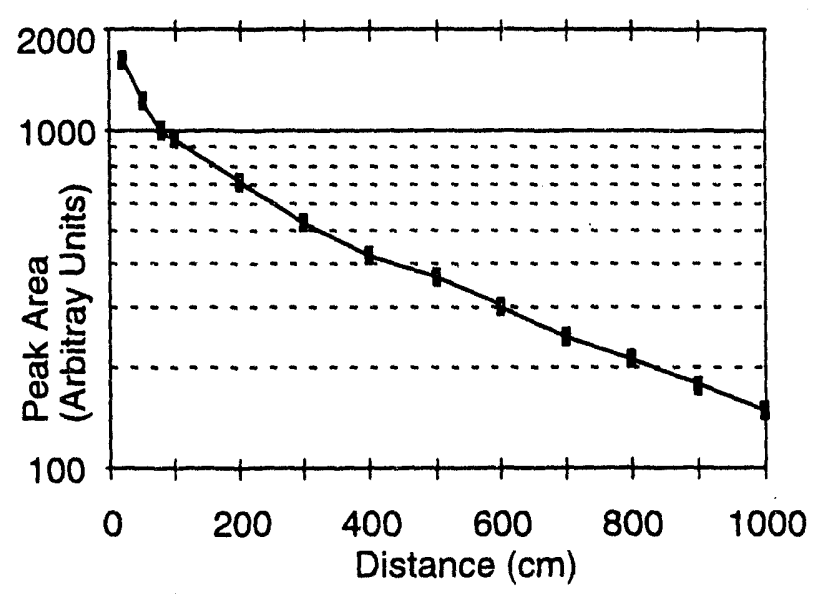

Figure 2. Results of transmission length measurements using UV-fluorescence excitation.

The transmission length is computed from the integrated intensities at 20 and $80 \mathrm{~cm}$ from the spectrometer. Although the attenuation is not perfectly linear in this region, it is a useful engineering measurement for detector design. The validity of this method for the application has been confirmed by nuclear measurements performed on fiber ribbons.
a GE615 silicone resin, for example.
b Guidedwave, Inc. 


\section{AGING}

Aging in these fibers is a phenomenon in which the transmission of the fiber degrades with time (Figure 3). Over about the first week a typical fiber loses about $30 \%$ of its transmission when stored in room air. The degradation of transmission length continues more slowly over a period of about 3 months until it stabilizes in the range of 35 to $50 \mathrm{~cm}$.

The value of the fibers as sensor materials would be greatly enhanced if their physical properties were stable with time. Although the transmission length is essentially constant after one month in room air, its final value is disappointingly low when compared to the initial value. Because the useful fiber length and, therefore, sensor area are determined by the attenuation length, it is desirable to maintain the greatest value possible.

\section{Aging Model}

Our model for aging is that atmospheric moisture diffuses through the silicone and corrodes the surface of the glass fiber at the silicone/glass interface. This corrosion layer scatters light, thereby reducing transmission. This model can be contrasted with alternatives, such as a change in the oxidation state of the cerium or changes in the silicone cladding.

Considerable experimental evidence supporting this conceptual model has been accumulated. Early confirmation came from the following observations: 1) substituting lanthanum for cerium did not significantly alter the aging behavior, 2) aging is slowed by anhydrous storage, 3) aging is accelerated by wet storage, and 4) large coiled bundles of fibers age more slowly than individual fibers (fibers act as a desiccant).

Other experiments and observations which add support to the aging model are: 1) silicone-clad, fused-silica fibers which have become fragile from aqueous corrosion have reduced transmission, 2) silicone-clad, fused-silica fibers which have not become fragile with time have about the same transmission as when they were made, and 3) attenuation measurements performed over short distances show faster aging than those performed over long distances on the same fiber. ${ }^{3}$

\section{Transmission Length is Affected by Solvents}

If an aged fiber is exposed to acetic acid, the transmission length increases. Several experiments were performed to clarify this phenomenon (Figure 4). Aged fibers were exposed to a variety of solvents. Some, such as hexane, had low refractive indices and were chemically inert. Others had a range of chemical activity from weakly polar (silicone resin) to highly polar (formic acid). Some were anhydrous, others were diluted with water. Hexane caused only a small improvement in transmission length, and eventually damaged the silicone coating by causing it to swell. Anhydrous, low molecular weight acids caused the biggest improvements in transmission length. The improvements in transmission length lasted only as long as the fibers were exposed to a saturated atmosphere or solution of the given solvent. This series of experiments indicated that something more complex than alteration of the refractive index of the cladding is occurring when the more reactive fluids are applied.

Freshly made fibers were exposed to glacial acetic acid, and the fluorescence peak area and transmission length were monitored. In the first few seconds, the transmission begins to increase and then drops to approximately the value of aged fiber. Then, over the period of a few minutes, 
the transmission increases to a valuc greater than that of the fresh fiber. The transmission then decreases as the fiber is allowed to dry. Within three days, the transmission length degrades to a

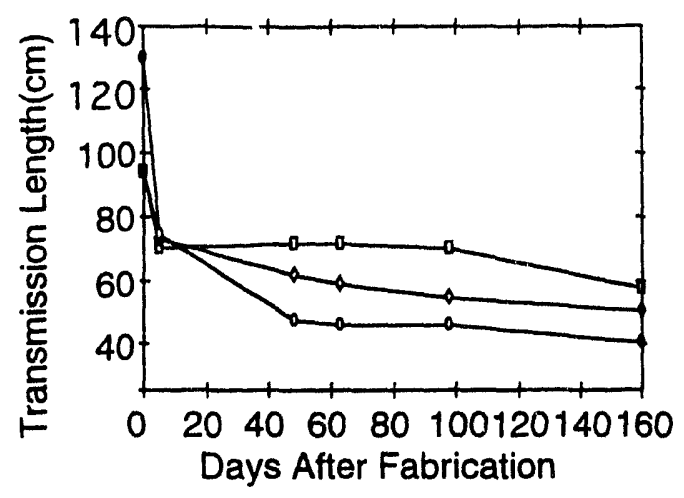

Figure 3. Aging in 3 typical silicone-clad lithium silicate fibers.

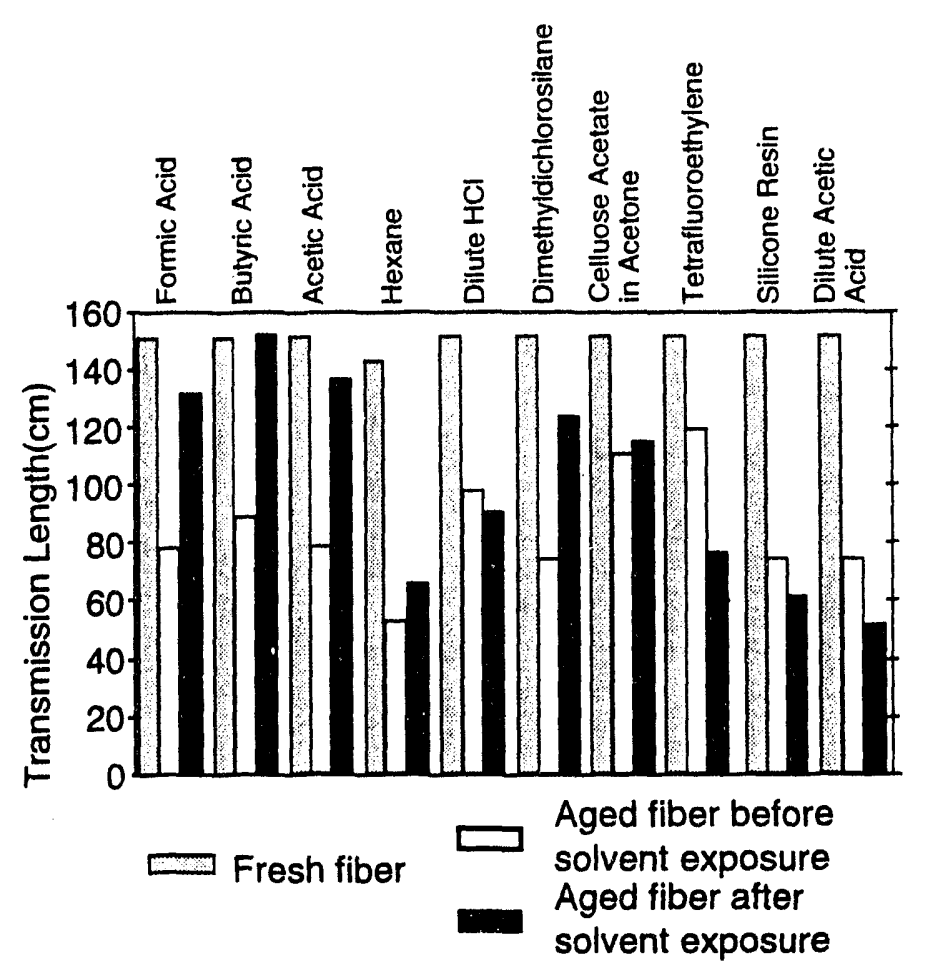

Figure 4. Effect of various liquids on fiber transmission length after the fibers were allowed to age in air for several weeks.

lower value than when it was first made. The changes in the transmission length are accompanied by similar increases and decreases in the fluorescence peak area. The integrated fluorescence peak area detected at the end of the fiber approximately doubles with acetic acid treatment regardless of fiber length. This indicates that the increase in transmission length is accompanied by more efficient light capture by the fiber. The reduction in scattering was confirmed by monitoring light scattered out of a fiber into an integrating sphere before and after exposure to glacial acetic acid. The intensity of the scattered light collected by the integrating sphere was halved after the fiber was exposed to glacial acetic acid.

Various scenarios of air and desiccator storage combined with exposures to glacial acetic acid and water consistently indicated that water degraded fiber transmission and that glacial acetic acid would recover at least some of the transmission.

The following model was developed to explain how the acetic acid reverses fiber aging. The acetic acid first acts to increase the corrosion of the fiber surface; the acid then reacts with the corrosion layer by converting the corrosion products to acetates. Eventually, the entire surface reacts to form a uniform acetate gel which scatters much less light than the original glass/silicone interface. The gel may also act as a graded index layer between the glass and silicone.

The amount of acetic acid involved need not be large. The acetic acid evolved in curing of RTV® (air-curing silicone) has been found to be sufficient to prevent aging if it is kept from evaporating. 
One-meter-long fibers were coated with RTV ${ }^{\circledR}$ and then encased in Gella ${ }^{\circledR}$, a leaving $\sim 15 \mathrm{~cm}$ of uncoated fiber at each end. The fibers were monitored for over 24 months. The transmission length was determined by measuring the integrated fluorescence peak intensity by exciting the fiber as it exited or entered the RTV and Gella sandwich (Figure 5). Bonding the fiber detector panels with RTV and then encasing the panels between sheets of Gella ${ }^{\circledR}$ has become the new standard for encapsulation. The principal disadvantage with such an approach is that it results in a bulky package; this can limit the possible applications of the technology.

\section{GLASS-ON-GLASS}

In an attempt to cure the aging problem without the use of RTV or Gella, a glass-on-glass sensor package development effort was initiated. A second platinum bushing was designed and fabricated to fit inside the existing bushings. The core/cladding glass ratio was estimated by assuming that laminar flow conditions prevail during drawing. The drawing process is similar to that for the single-crucible. The induction coil heats the outer bushing; heating of the inner bushing is via thermal conduction through the glass and the wall of the inner bushing.

The glass-on-glass fiber draw used a core glass containing added alumina and a cladding glass with one third of the lithium replaced by sodium. This composition was predicted to provide sufficient refractive index contrast and, therefore, the numerical aperture to result in guiding. This core and cladding combination was drawn with two different buffers: a high-refractive-index silicone buffer (Shinetsu OF20), and the standard low-refractive-index buffer (GE 615). It was also drawn with no buffer. The high-index buffer guarantees that the glass/silicone interface does not form a waveguide; all waveguiding occurs at the core/cladding interface.

The transmission length, when fresh, was $100 \mathrm{~cm}$ with the high-index buffer, $115 \mathrm{~cm}$ with the low-index buffer, and $130 \mathrm{~cm}$ with no buffer coat. The peak area, a measure of the fraction of the fluorescence trapped, of the fiber with the high-index buffer was only about $25 \%$ of that of the fiber with the low-index coating. The high-index buffered fibers did not demonstrate significant aging. This is confirmation of the model that corrosion at the optical interface is the cause of aging. The fibers buffered with low-index material showed some aging but slightly less than that which is usually experienced; this is interpreted as aging at the glass/silicone interface but not at the glass/glass interface. The uncoated fibers show initially superior transmission length compared to both the coated fibers; however, this value quickly degrades to nearly the same value as the highindex coated fibers. This is interpreted as accelerated aging of the air/glass interface with minimal aging at the glass/glass interface (Figure 6).

\section{SUMMARY}

Silicone-clad lithium silicate fibers exhibit, over time, a reduction in transmission. The aging studies and the effect of various solvents on fiber transmission clearly demonstrate that commercial silicone coatings are highly permeable to water and low molecular-weight organics. All the evidence is consistent with aging being the result of fiber surface corrosion by atmospheric water diffusing through the silicone.

Small amounts of acetic acid are sufficient to control or reverse the aging process. The acetic acid evolved in a package composed of RTV and a vapor barrier (Gella) has proven to stabilize the fiber performance for more than two years. This is satisfactory for most applications but does restrict the applications slightly because of the required bulk.

a $4441 / \mathrm{Gella}{ }^{\circledR}$ is a re-enterable encapsulant manufactured by $3 \mathrm{M}$ and is used to keep underground telephone cables dry. 
The aging data indicate that the glass-on-glass waveguides performed as expected. The aging data collected on the glass-on-glass fibers help to confirm the model which ties aging to corrosion of the glass surface.

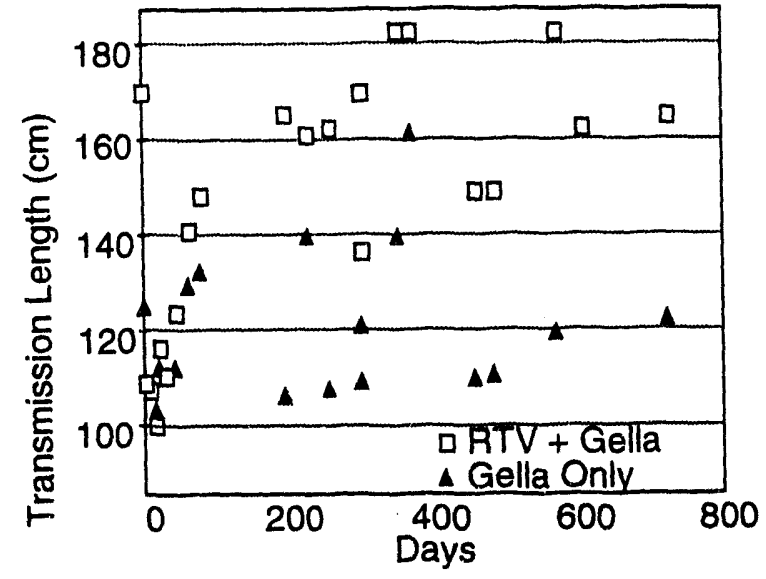

Figure 5. Variation of transmission length, over time, of fiber packages incorporating RTV silicone with a water barrier (Gella) and using Gella alone. Some of the scatter in the data is the result of using only two points for fluorescence peak area measurements. (The RTV and Gella are opaque to UV illumination.)

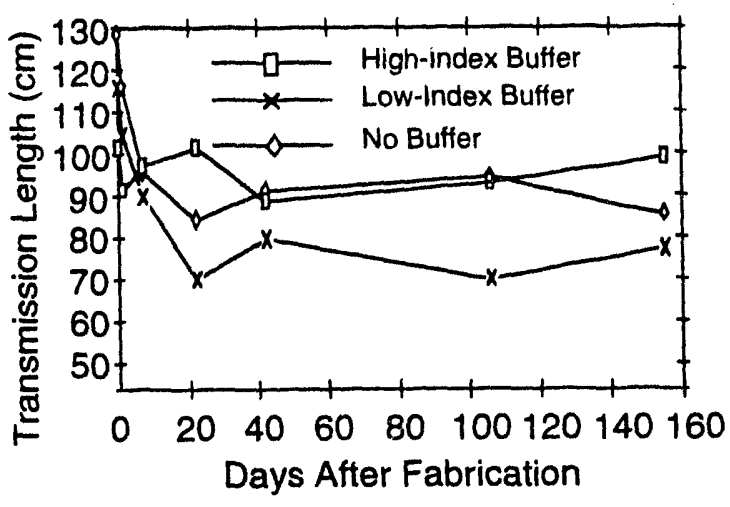

Figure 6. Change in transmission length with time for glass-on-glass scintillating fibers with and without high- and lowrefractive-index buffers.

In the most successful glass-on-glass fiber produced to date, the core/claddirg interface trapped approximately $25 \%$ as much light as the silicone/glass interface, based on peak areas. This small numerical aperture of the glass-on-glass fibers means that even the best fiber produced to date captures only one quarter of the light captured by a polymer-clad fiber. This large difference in capture efficiency means that, for many applications, an aged polymer-clad fiber would outperform a glass-on-glass fiber.

\section{ACKNOWLEDGEMENTS}

This work was supported, in part, by the Department of Energy (DOE), NN-20. Pacific Northwest Laboratory is operated by Battelle Memorial Institute for the DOE under contract DEAC06-76RLO 1830.

\section{REFERENCES}

1. G.B. Spector, et al., Nucl. Inst. Meth. 326, 526 (1993).

2. M. Atkinson, et al., Nucl. Inst. and Meth. A254, 500 (1987).

3. K. H. Abel et al.; Preceedings of SciFi93: Workshop on Scintillating Fiber Detectors;

October 24-28, 1993; Notre Dame, IN; World Press, in press. 

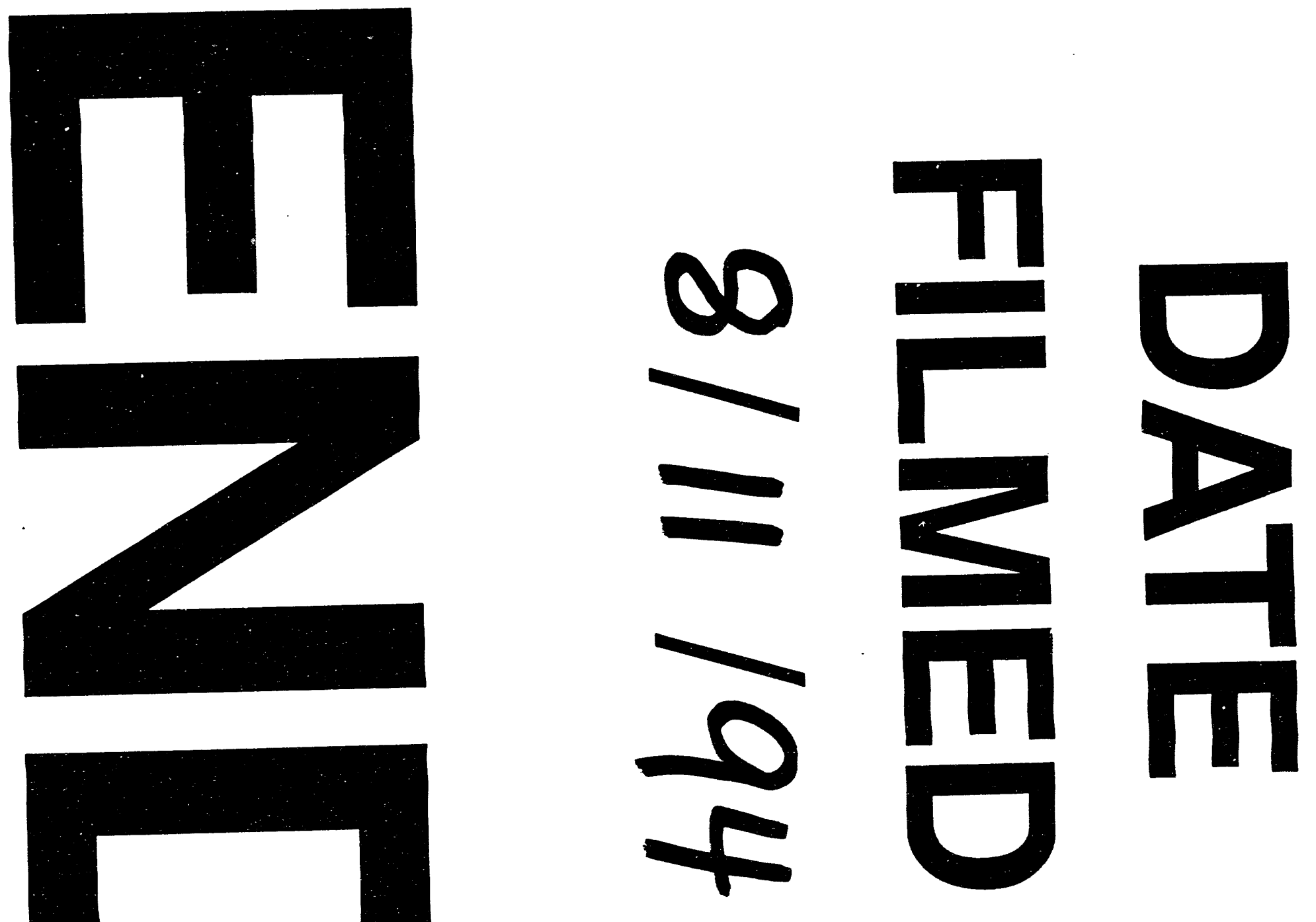
 